\title{
The electric double layer at high surface potentials: The influ- ence of excess ion polarizability
}

\author{
Marius M. Hatlo ${ }^{1}$ René van RoiJ ${ }^{1}$ And Leo LuE ${ }^{2}$ \\ 1 Institute for Theoretical Physics, Utrecht University, Leuvenlaan 4, 3584 CE Utrecht, The Netherlands \\ 2 Department of Chemical and Process Engineering, University of Strathclyde \\ James Weir Building, 75 Montrose Street, Glasgow G1 1XJ, United Kingdom
}

PACS $82.45 . \mathrm{Gj}$ - Electrolytes

PACS $73.30 .+\mathrm{y}-$ Surface double layers, Schottky barriers, and work functions

PACS 82.47. Uv - Electrochemical capacitors; supercapacitors

\begin{abstract}
By including excess ion polarizability into Poisson-Boltzmann theory, we show that the decrease in differential capacitance with voltage, observed for metal electrodes above a threshold potential, can be understood in terms of thickening of the double layer due to ion-induced polarizability-holes in water. We identify a new length which controls the role of excess ion polarizability in the double layer, and show that when this is comparable to the size of the effective Debye layer, ion polarizability can significantly influence the properties of the double layer.
\end{abstract}

Near a charged surface in contact with an electrolyte, attracted counterions and depleted coions form a layer that electrically screens the interfacial charge, rendering the so-called double layer electrically neutral. The counterion layer is loosely associated with the charged surface, under the influence of both the attractive and repulsive Coulomb force and thermal motion. The double layer has been studied extensively due to its importance within a wide number of areas e.g. colloid science, electrochemistry, and geophysics. Much attention has been devoted to the low and moderate surface potential regime, where established Gouy-Chapman-Stern (GCS) models [1-3] can quantitatively explain most experimental results. However, the double layer is also a key ingredient in applications such as supercapacitors [4] and microfluidic devices [5], which often operate at surface potentials much larger than the thermal voltage $\left(k_{B} T / e=25 \mathrm{mV}\right.$, where $e$ is the fundamental unit of charge). The behavior of double layers at such high voltages is not well understood, and experimentally available quantities, such as the electroosmotic mobility and the differential capacitance, deviate from established models based on GCS theory $[1-3,6]$. Many experimental observations in the high surface potential regime can be qualitatively explained by including ionic excluded volume interactions into Poisson-Boltzmann theory, either by a lattice approximation [6-8] or by the more accurate BMCSL equation of state $[6,9,10]$. However, quantitative agreement between theory and experimental data is in most cases only obtained with unphysically large ion sizes [6], suggesting that other effects are equally or more important.
In this Letter, we study ions in water near planar electrodes at a surface potential $\psi_{0}$, including not only excluded volume, but also the excess ion polarizbility, i.e. the change of the water polarizability due to the presence of an ion [11]. Due to excess ion polarizability, an additional dielectrophoretic force is exerted on the ions [12], which becomes comparable to the bare Coulomb force for large electric fields. The model without excluded volume interactions was first published in its present form in Ref. 11 and analyzed in more detail in Ref. 13. Similar models were used to describe excess polarizability effects in bulk electrolytes [14] and in double layers with charged polymers [15]. Studies of excess polarizability in the double layer dates back to the papers by Gouy [16], Frumkin [17], and Butler [18], who examined the influence of neutral molecules on the differential capacitance. The influence of excess ion polarizability on the differential capacitance was first studied by Bikerman [7]. The model used by Bikerman includes how excess ion polarizability changes the chemical potential of the ions, and due to lack of computational power, he restricted the study to the asymptotic behavior for weak and strong electric fields. It is also well known that excess polarizability also influence the dielectric constant [19], and a complete model of excess ion polarizability must capture both these effects, as done in Refs. [11,13]. The main result of this Letter is the finding that excess ion polarizability can explain the experimentally observed increase of the double layer at surface potentials larger than a certain threshold $\psi_{\alpha}$, for bulk packing fractions below $v_{0}$. We find an analytic expression for $\psi_{\alpha}$, and show that when 
$\psi_{0}>\psi_{\alpha}$ and $v<v_{0}$, the thickness of the double layer increases as a function of voltage due to an interplay between dielectrophoretic repulsion and Coulomb attraction. For $v>v_{0}$, the double layer thickness also increases with voltage, however, excluded volume interactions are the main driving mechanism, as found previously in Refs. 6-8. This increase of the double layer is qualitatively different from the decrease predicted by the standard GCS model and is in agreement with experimental measurements of the specific differential capacitance $C$

$$
C \equiv \frac{\partial \Sigma}{\partial \psi_{0}}
$$

of the double layer per unit area, where $\Sigma$ is the surface charge density of the electrode.

The first description of the double layer was due to Helmholtz [20], who considered it as a normal two plate capacitor: the metal electrode is separated from the electrolyte solution (assumed to behave as a perfect conductor) by a medium of dielectric constant $\epsilon$ and thickness $d$, due to the finite size of the ions. For a planar electrode, the capacitance per unit area according to the Helmholtz model is

$$
C^{H}=\frac{\epsilon}{4 \pi d},
$$

independent of $\psi_{0}$ and the bulk electrolyte concentration $c$.

In the early 1900's, Gouy [1] and Chapman [2] considerably improved this model by including the diffusive structure of the loosely bound counterions. The Gouy-Chapman differential capacitance, assuming symmetric monovalent ions (with charge $q_{ \pm}= \pm e$ ), is [1,2]

$$
C^{G C}=\frac{\epsilon}{4 \pi} \kappa \cosh \left(\frac{\phi_{0}}{2}\right) \equiv \frac{\epsilon}{4 \pi \lambda\left(\kappa, \phi_{0}\right)},
$$

where $\phi_{0}=\beta e \psi_{0}$ is the dimensionless electrode potential, $\kappa^{2}=8 \pi \beta e^{2} c / \epsilon$ is the Debye screening parameter, $\beta^{-1}=$ $k_{B} T, T$ is the temperature, and $k_{B}$ the Boltzmann constant. In analogy with the Helmholtz capacitance Eq. (2) $\lambda\left(\kappa, \phi_{0}\right)$ can be viewed as the effective thickness of the double layer, i.e. most of the neutralizing charge is located within a distance $\lambda\left(\kappa, \phi_{0}\right)$ from the electrode. The effective thickness of the double layer within the $\mathrm{GC}$ model $\lambda\left(\kappa, \phi_{0}\right)$ is a decreasing function of both $\psi_{0}$ and $c$, and approaches zero when $\left|\phi_{0}\right| \rightarrow \infty$. However, such unphysical divergence of the differential capacitance is not observed experimentally [21-23], and it is only when $\lambda\left(\kappa, \phi_{0}\right) \gg d$, corresponding to low surface potential and dilute electrolyte solutions, that this model accurately describes the double layer. For monovalent ions in water, ion-ion correlations (including image charges) play a minor role, justifiying the mean field approximation underlying the Gouy-Chapman model [24].

To describe the regime where $\lambda\left(\kappa, \phi_{0}\right) \sim d$, Stern suggested a combination of the Gouy-Chapman model and the Helmholtz model, splitting the double layer into a normal two-plate capacitor close to the electrode (Eq. (2)), in series with the GouyChapman capacitance (Eq. (3)) [3]. The GCS model is now the established way of describing the double layer, and can explain many of its aspects, especially at low to moderate voltage.
When the voltage is increased beyond a certain level (varies with electrolyte and electrode), the GCS model fails, e.g. it cannot explain the experimentally observed nonmonotonic dependence of the differential capacitance with increasing voltage $[21,25,26]$. Such nonmonotonic behavior is often interpreted in terms of a voltage dependent Stern capacitance. From fits of experimental measurements to such a model, it has been concluded that the dielectric constant of the Stern layer is different from bulk water, and varies with voltage $[25,27]$.

The influence of static excess ion polarizability on the electric double layer has been studied in various forms, dating back to the classic paper by Bikerman [7]. In recent years, a modified Poisson-Boltzmann approach has been developed, which consistently takes into account excess ion polarizability $[11,13]$, describing both its influence on the chemical potential of the ions and the dielectric constant of the solution. These studies suggest that excess ion polarizability can significantly influence the double layer when $\left|\phi_{0}\right| \gg 1$. It is experimentally known that the dielectric constant of a bulk electrolyte varies linearly with bulk salt concentration for $c<2 \mathrm{M}$ [19]. Within a mean field approximation, this linear relation also holds for an inhomogeneous system. The net dielectric constant of the solution then becomes position dependent, and at a distance $z$ from a planar electrode, $[11,13,14,19]$

$$
\epsilon(z)=\epsilon_{w}+4 \pi \alpha_{+} c_{+}(z)+4 \pi \alpha_{-} c_{-}(z),
$$

where $\epsilon_{w}$ is the dielectric constant of water, $c_{ \pm}(z)$ is the local concentration of cations $(+)$ and anions $(-)$, and $\alpha_{ \pm}$is the excess polarizability of the ions. In Eq. (4), we assume the dielectric constant of water to be equal to its bulk value, valid for electric fields below $0.25 \mathrm{~V} \mathrm{~nm}^{-1}$ [27-30]. For most ions in water $\alpha<0$ [19], meaning they reduce the dielectric constant of the solution. The measured values of the excess ion polarizability are tabulated in Table 1 . Note that the actual polarizability of the ion is not the most important, but rather the polarizability hole that the ion creates in water [13,31]. The negative sign of $\alpha$ can be further understood by invoking the Clausius-Mossotti relation [30], which for a homogenous dielectric constant $\epsilon_{i}$ inside the polarizability hole is given by

$$
\alpha=R_{i}^{3} \epsilon_{w} \frac{\epsilon_{i}-\epsilon_{w}}{\epsilon_{i}+2 \epsilon_{w}}
$$

where $R_{i}$ is the effective ion radius, including the water molecules in the hydration shell. With $\epsilon_{i}<\epsilon_{w}$, due to the orientationally restricted water molecules in the hydration shell, we have $\alpha<0$.

Within a mean field approximation, the excess energy of a polarizable particle in an external electric field is $[7,11,13,14$, 19]

$$
\beta E_{\mathrm{ex}}=\frac{1}{2} l^{2}\left|\phi^{\prime}(z)\right|^{2}
$$

where the prime denotes a derivative, and $l=\left(-\alpha / \beta e^{2}\right)^{1 / 2}$ is a new length scale characterizing the coupling between bare and induced charges (we assume equal $\alpha$ for both ion species for notational convenience). The quadratic relation in Eq. (6) was found in Ref. 30 to hold for $\left|\psi^{\prime}\right|<0.25 \mathrm{~V} \mathrm{~nm}^{-1}$; for 


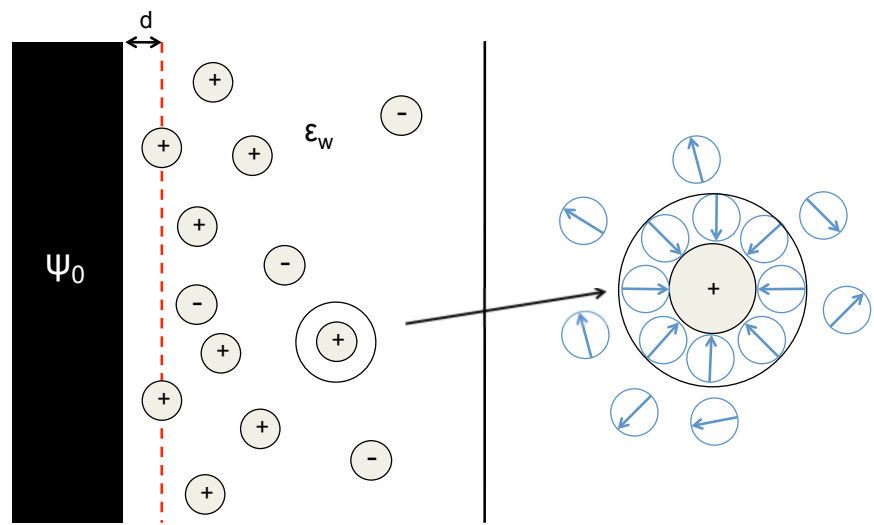

Fig. 1: Electrode at potential $\psi_{0}$ in contact with an electrolyte solution. The ions are restricted from within a distance $d$ from the plate and are surrounded by a layer of ordered water molecules (see Ref. 13 for a similar illustration).

$\left|\psi^{\prime}\right| \gg 0.25 \mathrm{~V} \mathrm{~nm}^{-1}$ the energy becomes linear in the electric field due to the polarizability saturation of water [7]. In this Letter, we do not include dispersion interactions between the ions and the electrode; such interactions are very short ranged and are approximately captured here by the thickness of the inner Stern layer.

To estimate the double layer thickness $\zeta$ for $\left|\phi_{0}\right| \gg 1$, we compare the induced-dipole contribution Eq. (6) with the term due to bare charges, $l^{2}\left|\phi^{\prime}(0)\right|^{2} / 2=\phi_{0}$. Assuming the potential varies linearly through the double layer, $\phi^{\prime}(0) \approx \phi_{0} / \zeta$, it follows

$$
\zeta \sim l \sqrt{\left|\phi_{0}\right|},
$$

which is an increasing function of the surface potential, in contrast to $\lambda\left(\kappa, \phi_{0}\right)$. Below, in Eq. (12), a more detailed expression for $\zeta$ will be derived.

The geometry we study, similar to a typical experimental setup, is shown in Fig. 1. An electrolyte solution with bulk ion concentration $2 c$ is in contact with a planar electrode at constant potential $\psi_{0} \equiv \psi(z=0)$, and the solvent is modeled as a continuum with dielectric constant $\epsilon_{w}$. The ions are restricted from a region of thickness $d$ near the electrode, corresponding to the inner Stern/Helmholtz layer.

By including the induced dipole density of the ions in the Poisson equation, we find [32]

$$
-\frac{1}{4 \pi}\left\{\epsilon(z) \psi^{\prime}(z)\right\}^{\prime}=e\left(c_{+}(z)-c_{-}(z)\right)
$$

where $\epsilon(z)$ is the effective dielectric constant given by Eq. (4).

To complete Eqs. (8) and (4), we relate the local electric potential to the local ion density by the Boltzmann distribution $[7,13,14]$. To include excluded volume interactions, we use the lattice approach introduced by Bikerman in Ref. 7

$$
c_{ \pm}(z)=\frac{c \exp \left[\mp \phi(z)-\frac{l^{2}}{2}\left|\phi^{\prime}(z)\right|^{2}\right]}{1+v \exp \left[-\frac{l^{2}}{2}\left|\phi^{\prime}(z)\right|^{2}\right] \cosh (\phi(z))-v},
$$

Table 1: Experimentally measured excess polarizability $[13,19]$.

\begin{tabular}{lr|lr}
\hline \hline cation & $4 \pi \alpha\left(\mathrm{M}^{-1}\right)$ & anion & $4 \pi \alpha\left(\mathrm{M}^{-1}\right)$ \\
\hline $\mathrm{H}^{+}$ & -17 & $\mathrm{~F}^{-}$ & -5 \\
$\mathrm{Li}^{+}$ & -11 & $\mathrm{Cl}^{-}$ & -3 \\
$\mathrm{Na}^{+}$ & -8 & $\mathrm{I}^{-}$ & -7 \\
$\mathrm{~K}^{+}$ & -8 & $\mathrm{OH}^{-}$ & -13 \\
$\mathrm{Rb}^{+}$ & -7 & $\mathrm{SO}_{4}^{-}$ & -7 \\
\hline \hline
\end{tabular}

where $v=2 c a^{3}$, and $a$ is the lattice spacing, approximately equal to the ion diameter. Ions that increase the local dielectric constant of the solution are attracted to regions of high fields, while ions that decrease the dielectric constant are repelled. The extra repulsion/attraction is caused by the dielectrophoretic force due to polarization effects in a nonuniform electric field [12]. Assuming a uniform dielectric constant in the Stern layer, the electric potential obeys

$$
\psi(d)=\psi_{0}+d \psi^{\prime}(d) .
$$

In Fig. 2a, we plot the ion density profile (solving Eqs. (4), (8) and (9)) for $\phi_{0}=15,4 \pi \alpha=-8 \mathrm{M}^{-1}, a=0.6 \mathrm{~nm}$ and $d=0$ at different bulk ion concentrations. We observe three surprising phenomena: (i) a saturation of the density profile approaching $-8 \pi \alpha c(z) / \epsilon_{w}=1$, (ii) an increase of the thickness of the saturated layer with concentration $c$, completely opposite to the established GCS model, and (iii) almost identical density profiles for $a=0.0 \mathrm{~nm}$ and $a=0.6 \mathrm{~nm}$. The ion concentration close to the plate saturates at $c \approx 5 \mathrm{M}$, which for an ion of hydrated diameter $a=0.6 \mathrm{~nm}$ corresponds to $v \approx 0.65$, i.e. below close packing. To facilitate a simple analysis to better understand the physics involved, we assume zero coion density in the vicinity of the electrode. Without any loss of generality, we assume negatively charged counterions, i.e. $\phi_{0}>0$. For the planar geometry depicted in Fig. 1, with $\phi_{0} \gg 1$ there is an analytical solution to Eqs. (8), (4), and (9) for $\alpha<0$ and $z<\zeta$ [13],

$$
\phi_{0}-\phi(z)=\frac{z}{2 l^{2}}[2 \zeta-z]
$$

where

$$
\zeta=l \sqrt{2\left[\phi_{0}-\phi_{\alpha}\right]}
$$

which is a more accurate version of Eq. (7). The solution is only valid for $\phi_{0}>\phi_{\alpha}=\ln \left([1-v] /\left[(\kappa l)^{2}-v / 2\right]\right),(\kappa l)^{2}>$ $v / 2$, and $v<1$. For $(\kappa l)^{2}<v / 2$ saturation of the density profile due to excluded volume will dominate, a regime which has been analyzed elsewhere [6]. For the polarizability of $\mathrm{K}^{+}$, excluded volume will dominte the large potential regime when $a>0.7 \mathrm{~nm}$. As predicted earlier, $\zeta$ is an increasing function of the surface potential, and, as suggested by Fig. $2 \mathrm{a}, \zeta$ increases with concentration $c$.

The resulting counterion density is $c_{-}(z)=c /(\kappa l)^{2}=$ $-\epsilon_{w} / 8 \pi \alpha$, the effective dielectric constant is $\epsilon=\epsilon_{w} / 2$ (see Eq. (4)), and the differential capacitance per unit area is

$$
C=\frac{\epsilon}{4 \pi \zeta}=\frac{\epsilon_{w}}{8 \pi \zeta}
$$




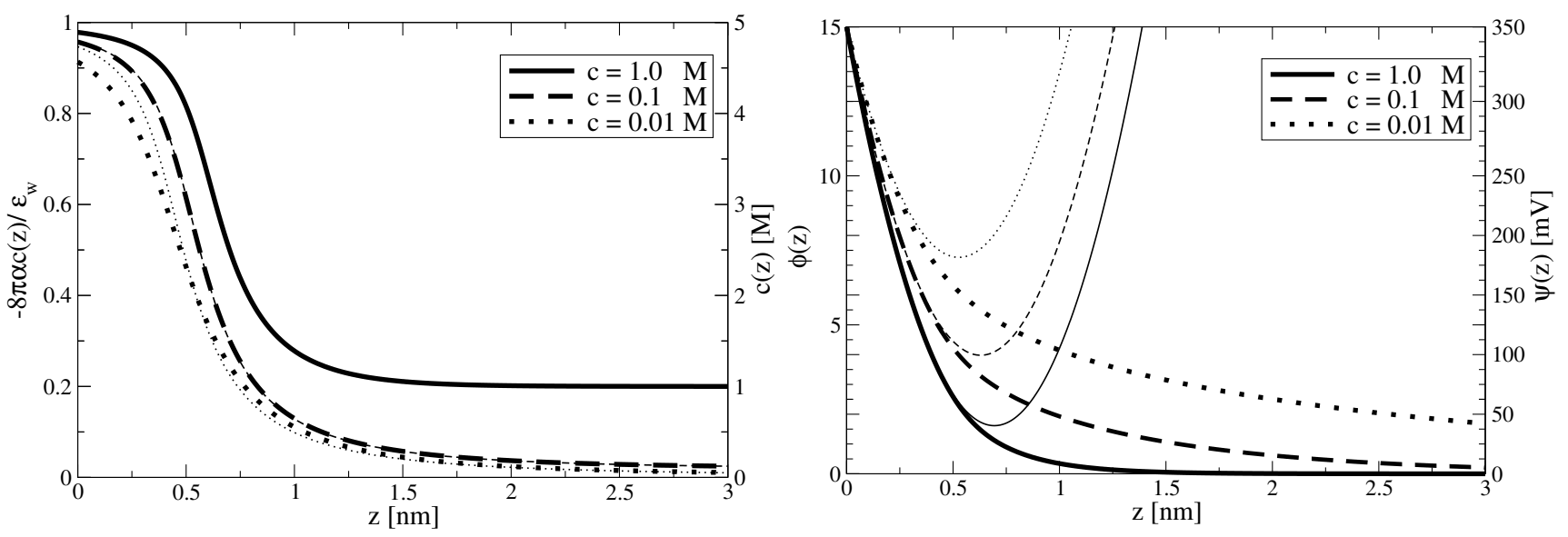

Fig. 2: (a) Counterion density profiles and (b) electric potential near an electrode at $\beta e \psi_{0}=15$ for $4 \pi \alpha=-8 \mathrm{M}^{-1}$ (corresponding to $\mathrm{K}^{+}$), $a=0.6 \mathrm{~nm}$ and different bulk ion concentrations. The thin lines in (a) are with $a=0.0 \mathrm{~nm}$ and in (b) the analytical approximation Eq. (11).

confirming that $\zeta$ is the effective thickness of the double layer.

In Fig. 2b, we plot the electric potential for different bulk electrolyte concentrations, and compare the result with the analytical solution. The analytical solution is accurate for $z<\zeta$ and becomes unphysical for $z>\zeta$, where the potential increases with $z$.

In Fig. 3, we plot the electric field at the electrode surface $\left(E(0)=-\psi^{\prime}(0)\right)$ as a function of the surface potential $\psi_{0}$, for different values of the bulk electrolyte concentration $c$. The excess ion polarizability are for both ions set equal to $\mathrm{K}^{+}$in Table 1 . Here we see that the maximum electric field in the regime of interest is below the limit where the polarization saturation of water must be accounted for $\left(E(0)<0.25 \mathrm{~V} \mathrm{~nm}^{-1}\right)$. We also see from Fig. 3 that the field with and without excluded volume interactions are nearly identical, signifying the relative importance of ionic excess polarization compared to ionic packing.

In Fig. 4a, we compare the differential capacitance calculated numerically to the low voltage result given by Eq. (3) and the high voltage result given by Eq. (13). For intermediate voltages both approximations fail, and a full numerical solution must be used to obtain accurate results. To validate our theoretical predictions we compare the results with experimental data for the differential capacitance of $\mathrm{KPF}_{6}$ in contact with a silver electrode [21]. Neglecting the Stern layer (i.e. $d=0$, see Fig. 1), the agreement between theory and measurments is only qualitative (thick dashed-dotted line in Fig. 4a), and the differential capacitance is overestimated. Good agreement between our theory and the experimental data is found for $d=4.8 \AA$ corresponding to $C^{H}=146 \mu \mathrm{F} \mathrm{cm}^{-2}$ (solid line of Fig. 4a), in agreement with values obtained in previous studies $[6,33]$. In Fig. $4 b$, we compare our results with the same measurements for several bulk ion concentrations, with $d=4.8 \AA$ and excess ion polarizabilities for both ions corresponding to $\mathrm{K}^{+}$in Table 1 . The agreement is very good, especially for negative voltage, when $\mathrm{K}^{+}$is the counterion. To obtain similar agreement for positive voltage, it is necessary to use a different ion polarizability for $\mathrm{PF}_{6}^{-}$, as well as two different distances of closest approach, as the ions have different effective sizes in water.

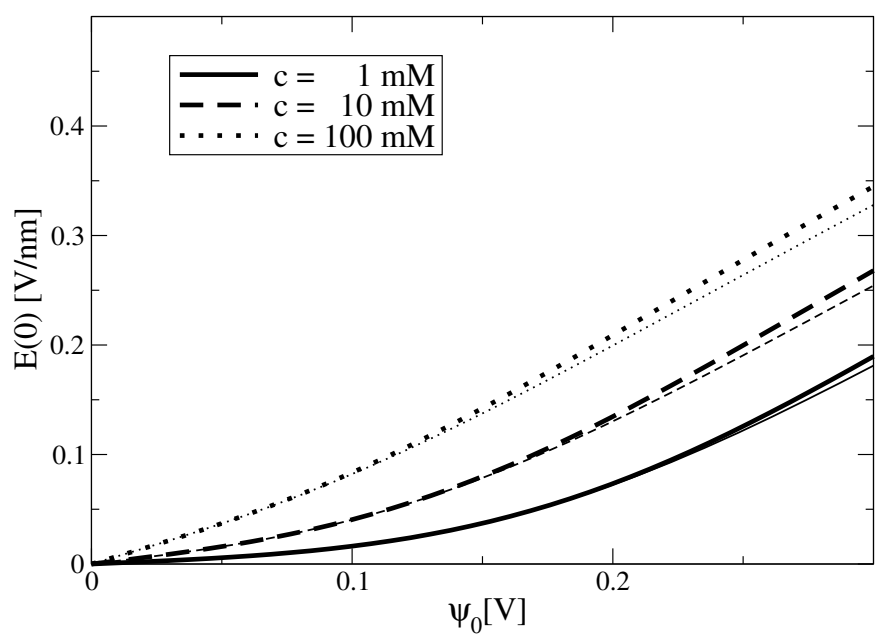

Fig. 3: The maximum electric field in the double layer as a function of surface potential for $4 \pi \alpha=-8 \mathrm{M}^{-1}$ and $d=0.48 \mathrm{~nm}$. Thick lines with $a=0.6 \mathrm{~nm}$ and thin lines with $a=0.0 \mathrm{~nm}$.

In Fig. 5, we plot the counterion density a distance $d$ from the electrode surface as a function of the surface potential $\psi_{0}$, for different values of the bulk electrolyte concentration $c$. The excess ion polarizability are for both ions set equal to $\mathrm{K}^{+}$in table 1. In the regime of interest the maximum ion density is below $4 \mathrm{M}$, which is in the regime where Eq. (4) is valid [19]. The thin lines are the predictions with $a=0.6 \mathrm{~nm}$ corresponding to an ion radius of $R=0.3 \mathrm{~nm}$, which is about the hydrated ion radius of $\mathrm{K}^{+}$. Excluded volume interactions reduce the surface density, however only in a small quantitative manner. In Fig. 6 we plot the capacitance with a lattice spacing $a=0.6 \mathrm{~nm}$. As we can see from Fig. 6 the excluded volume interactions do not change the results much compared to the $v=0$ (see Fig. 4).

We find similar agreement between our theory and measurements of the differential capacitance at other electrodes [22,23], however with a different inner Stern layer thickness $d$ and capacitance $C^{H}$, suggesting that these quantities are electrode 

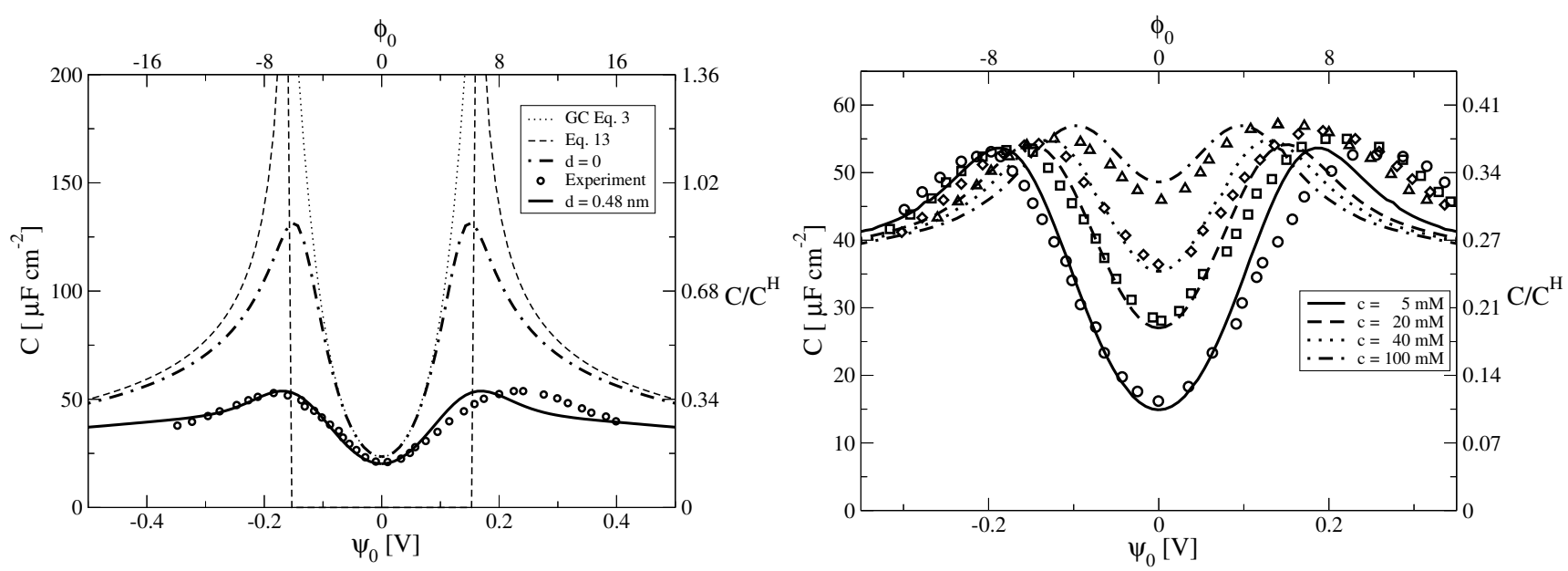

Fig. 4: Experimentally measured [21] differential capacitance (symbols) of an Ag-electrode in an aqueous $\mathrm{KPF}_{6}$ solution, in (a) for a salt concentration $c=0.01 \mathrm{M}$ and in (b) for a series of $c$ 's. The curves are theoretical predictions with $4 \pi \alpha=-8 \mathrm{M}^{-1}$ (for $\mathrm{K}^{+}$), $a=0.0 \mathrm{~nm}$, and a single fit parameter $d=0.48 \mathrm{~nm}$. In (a) the vertical lines represent the threshold potential $\pm \psi_{\alpha}$, the dot-dashed curve is the prediction for $d=0$, and the dotted and dashed curves are the asymptotic low- and high-potential curves of Eqs. (3) and (13), respectively.

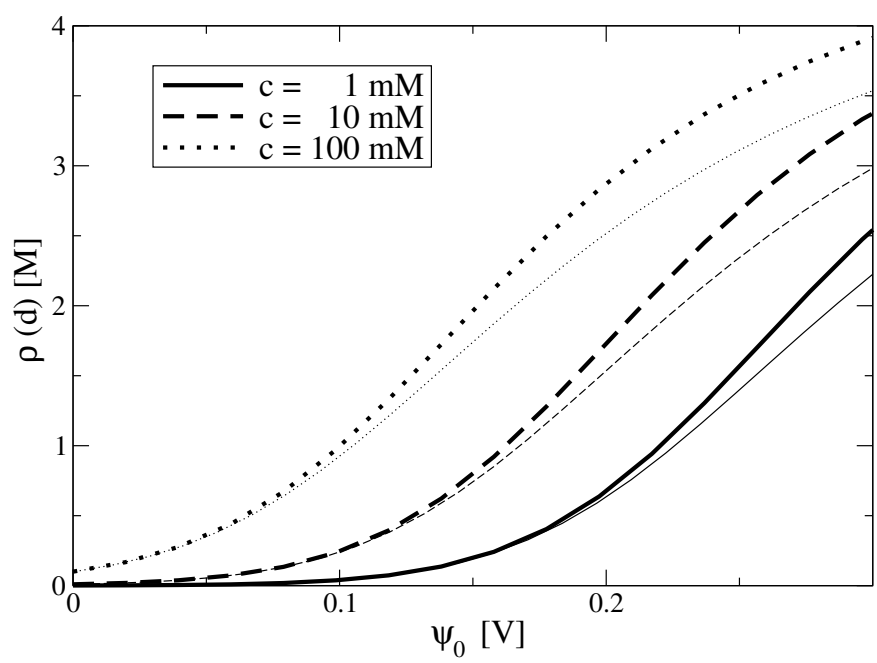

Fig. 5: The counterion density at the distance of closest approach as a function of surface potential for $4 \pi \alpha=-8 \mathrm{M}^{-1}$ and $d=0.48 \mathrm{~nm}$. The thick lines are the results without excluded volume and the thin lines are with excluded volume, with $a=0.6 \mathrm{~nm}$.

specific, and reflect interactions between water and the electrode material.

In this Letter, we have shown that excess ion polarizability strongly affects the double layer at large surface potentials. There is a decrease in capacitance as a function of voltage and bulk ion concentration above a threshold voltage $\psi_{\alpha}$. This trend is opposite to the well-known behavior at thermal voltages. When we account for the inner Stern layer, quantitative agreement between experimental measurements and our theory is found. For surface potentials above $\psi_{\alpha}$, the double layer thickens due to dielectrophoretic repulsion of ions from the charged surface. We have shown that saturation of the ion density profile near the electrode due to excess ion polarizability will for

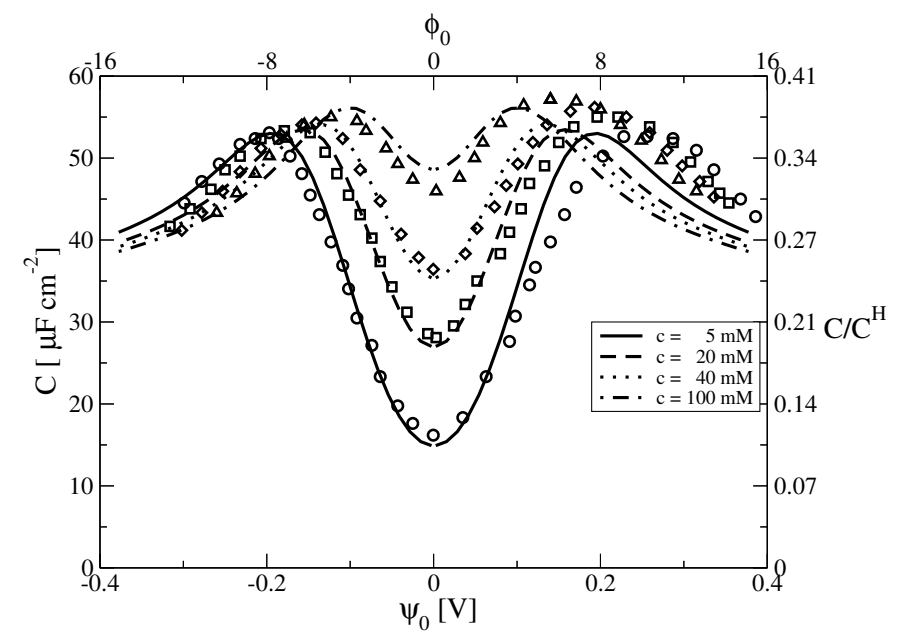

Fig. 6: Experimentally measured [21] differential capacitance (symbols) of an Ag-electrode in an aqueous $\mathrm{KPF}_{6}$ solution. The curves are theoretical predictions with $4 \pi \alpha=-8 \mathrm{M}^{-1}, a=0.6 \mathrm{~nm}\left(\right.$ for $\mathrm{K}^{+}$), and a single fit parameter $d=0.48 \mathrm{~nm}$.

many systems occur before saturation due to excluded volume, and will therefore be more important. Our new contribution to the theory of the double layer will facilitate a better understanding of supercapacitors at high surface potentials, used for example in desalination and blue energy devices [34-36], and for microfludic devices based on induced charge electroosmotic flow [6].

The theory presented in this Letter is valid when the dielectric constant of water is nearly unaffected by the external electric field, which is found in Ref. 30 to hold for $E<$ $0.25 \mathrm{~V} \mathrm{~nm}^{-1}$. Above this limit, the influence of the electric field on the water structure must be accounted for $[30,37]$. Including the polarizability saturation of water [27] leads to a decrease of the differential capacitance with voltage, in the same 
manner as predicted here, and could thus provide an alternative for the presently proposed excess-polarization mechanism. However, this alternative relies on two or more fit parameters to explain the data of Ref. 21. This is in contrast to the prediction presented here, where no fit parameter is needed other than the thickness of the Stern layer $d$.

Correlation effects in electrolytes become important when $l_{B} / a \gg 1$, where the interaction between ions becomes much larger than the thermal energy. For the present system, $l_{B} / a \sim$ 1 , and correlation effects will only give a small quantitative correction, even at high ion density. For systems with ions of higher valency and/or solvents with lower dielectric constant, correlation effects must be accounted for $[38,39]$. For systems without a solvent (e.g., ionic liquids), the ion polarizability is positive and will have the opposite effect on the differential capacitance [40] than that found in this work.

In many electrolyte-electrode systems specific adsorption of anions plays an important role, and such behavior must be included to obtain quantitative agreement between theory and measurements. In order to disentangle fundamental processes we have therefore considered here an electrode-electrolyte system where little specific anion adsorption is found. In this work, the maximum electric field is $<0.25 \mathrm{~V} \mathrm{~nm}^{-1}$, where the dielectric constant of water is still nearly equal to its bulk value $[29,30]$. We therefore believe that including polarizability saturation of water into the present theory, will only lead to a small quantitative change that can be captured by adjusting $d$. However, for even larger voltages and/or other electrode materials, the influence of the electric field on the dielectric constant of water must be included, for example along the lines of the work by Grahame [25]. Specific interactions between the ions and the electrode material may be accounted for by incorporating additional contributions to the Boltzmann weights in the numerator and denominator of Eq. (9). The present work is a natural starting point for extensions along all these lines.

This work is part of the reasearch program of the "Stichting voor Fundamenteel Onderzoek der Materie (FOM)".

\section{REFERENCES}

[1] Gouy G., J. Phys. (France) , 9 (1910) 475.

[2] Chapman D. L., Philos. Mag. , 25 (1913) 475.

[3] Stern O. Z., Electrochem., 30 (1924) 508.

[4] JaYALAKShmi M. and Balasubramanian K., Int. J. Electrochem. Sci., 3 (2008) 1196.

[5] Stone H., Stroock A. and Ajdari A., Annu. Rev. Fluid Mech. , 36 (2004) 381411

[6] Bazant M. Z., Kilic M. S., Storey B. D. and Ajdari A., Adv. Colloid Interfac. Sci. , 152 (2009) 48.

[7] Bikerman J. J., Philosophical magazine , 33 (1942) 384.

[8] Kilic M. S., Bazant M. Z. and Ajdari A., Phys. Rev. E, 75 (2007) 021503.

[9] Lue L., Zoeller N. and Blankschtein D., Langmuir , 15 (1999) 3726.
[10] Biesheuvel P. M. and van Soestbergen M., J. Colloid Interf. Sci. , 316 (2007) 490.

[11] Ben-YaAkov D., Andelman D., Harries D. and PodGORNIK R., J. Phys.: Condens. Matter, 21 (2009) 424106.

[12] Pohl H. A., J. Appl. Phys. , 22 (1951) 869.

[13] Ben-YaAkov D., Andelman D. and Podgornik R., $J$. Chem. Phys., 134 (2011) 074705.

[14] Netz R. R., Eur. Phys. J. E, 5 (2001) 189.

[15] Biesheuvel P. M., Eur. Phys. J. E, 16 (2005) 353.

[16] Gouy G., Ann. de physique, 175 (1917) 129.

[17] Frumkin V. A., Z. f. Physik, 35 (1929) 792.

[18] Butler J. A. V., Proc. R. Soc. A, 122 (1929) 399.

[19] Hasted J. B., Ritson D. M. and Collie C. H., J. Chem. Phys., 16 (1948) 1.

[20] Helmholtz H., Pogg. Ann. , 211 (1853) .

[21] Valette G., J. Electroanal. Chem., 122 (1981) 285.

[22] Valette G., J. Electroanal. Chem., 138 (1982) 37.

[23] Grahame D. C., J. Am. Chem. Soc. , 76 (1954) 4819.

[24] Hatlo M. M. and Lue L., Soft Matter , 4 (2008) 1582.

[25] Grahame D. C., J. Chem. Phys. , 18 (1950) 903.

[26] Hamelin A. and Stoicoviciu L., J. Electroanal. Chem., 234 (1987) 93.

[27] Bоотн F., J. Chem. Phys. , 19 (1951) 391.

[28] Danielewicz-Ferchmin I. and Ferchmin A. R., Chem. Phys. Lett. , 351 (2002) 397.

[29] Joshi R. P., Qian J., Schoenbach K. H. and Schamiloglu E., J. Appl. Phys. , 97 (2004) 3617.

[30] Gavryushov S. and Linse P., J. Phys. Chem. B , 107 (2003) 7135.

[31] Netz R. R., Curr. Opin. Colloid Interfac. Sci. , 9 (2004) 192.

[32] JACKson J. D., Classical Electrodynamics (Wiley, New York) 1975.

[33] Beltramo G., Giesen M. and Ibach H., Electrochimica Acta, 54 (2009) 4305.

[34] Boon N. and van Roij R., Mol. Phys., 109 (2011) 1229.

[35] Biesheuvel P. M. and VAN Der Wal A., J. Membrane Sci. , 346 (2010) 256.

[36] Brogioli D., Phys. Rev. Lett., 103 (2009) 058501.

[37] Frydel D. and Oettel M., Phys. Chem. Chem. Phys., 13 (2011) 4109.

[38] Bazant M. Z., Storey B. D. and Kornyshev A. A., Phys. Rev. Lett. , 106 (2011) 046102.

[39] Hatlo M. M. and Lue L., Europhys. Lett. , 89 (2010) 25002.

[40] Lauw Y., Horne M. D., Rodopoulos T. and LeERMaKers F. A. M., Phys. Rev. Lett. , 103 (2009) 117801. 